\title{
Sintomas Urinários em Primíparas de Parto Normal e Cesárea
}

\section{Urinary Symptoms in Primiparous women of Normal and Cesarean Delivery}

\author{
Sandy Vieira Siqueira ${ }^{1}$ \\ Jéssica de Nazaré Rodrigues Souto ${ }^{1}$ \\ Cibele Nazaré Camara Rodrigues ${ }^{2}$ \\ Lucieny da Silva Pontes ${ }^{3}$ \\ Gustavo Fernando Sutter Latorre ${ }^{4}$ \\ Erica Feio Carneiro Nunes ${ }^{3}$
}

RESUMO

Objetivo: comparar os sintomas urinários de primíparas de parto normal e cesárea. Métodos: foi realizado um estudo observacional analítico do tipo transversal realizado com 98 primíparas O estudo ocorreu no Centro de Saúde Escola do Marco, da Universidade do Estado do Pará, na Cidade de Belém do Pará, com mulheres que fizeram o acompanhamento do pré-natal nos anos de 2012 e 2013. A amostra foi definida por conveniência. Os critérios de inclusão para a participação da pesquisa foram: mulheres com idade entre 18 e 35 anos, primíparas de parto normal ou cesárea no período de dois meses a dois anos do pós-parto e que tenham assinado o TCLE. Os critérios de exclusão foram: mulheres multíparas, que tiveram histórico de gravidez de risco, diabéticas, hipertensas, que estivessem em novo período gestacional ou tiveram período gestacional menor que 37 semanas e que apresentaram algum sintoma urinário prévio à gravidez. A análise estatística foi feita com aplicação do Teste Exato de Fisher com índice de significância de 5\%. Resultados: Participaram do estudo 98 mulheres primíparas, sendo que 41 primíparas de parto normal e 57 de parto cesárea. Quanto à prevalência dos sintomas urinários relacionados ao tipo de parto, observou-se que a noctúria foi citada por 13 $(22,8 \%)$, seguida da polaciúria $8(14 \%)$ das mulheres que realizaram parto cesárea, enquanto que a urge-incontinência e a polaciúria ocorreram em $13(31,71 \%)$ e $6(14,6 \%)$, respectivamente, mulheres que tiveram parto normal. Conclusão: 0 surgimento dos sintomas urinários independe da via de parto.

DESCRITORES: Sistema urinário. Sinais e sintomas. Parto Normal. Cesárea

\begin{abstract}
Objective: to compare urinary symptoms in primiparous women with normal and cesarean delivery. Methods: An observational, analytical cross-sectional, study was performed with 98 primiparous women. The study was carried out at the Center of Health School of Marco, of the State University of Pará, in the city of Belém do Pará, Brazil, with women who underwent antenatal care between the years of 2012 and 2013. Sampling was defined for convenience. The inclusion criteria for the participation of the research were: women aged between 18 and 35 years, primiparous women of normal birth or cesarean section in the period of two months to two years postpartum and who signed the ICF. The exclusion criteria were: multiparous women, who had a history of risky pregnancies, diabetic, hypertensive, who were in a new gestational period or had a gestational period of less than 37 weeks, and who presented some urinary symptom prior to the pregnancy. Statistical analysis was performed using Fisher's Exact Test with a significance level of 5\%. Results: 98 primiparous women participated in the study, 41 of whom were primiparous women of normal birth and 57 of cesarean birth. Regarding the prevalence of urinary symptoms related to the type of delivery, nocturia was cited by $13(22.8 \%)$, followed by polaciuria $8(14 \%)$ of the women who underwent cesarean delivery, whereas urge-incontinence and polaciuria occurred in 13 $(31.71 \%)$ and $6(14.6 \%)$, respectively, women who had normal delivery. Conclusion: The appearance of urinary symptoms does not depend on the route of bith delivery.
\end{abstract}

DESCRIPTORS: Urinary system. Signals and symptons. Normal birth. Cesarean section.

\footnotetext{
. Graduanda do curso de Fisioterapia. Universidade da Amazônia- UNAMA. Belém, Pará, Brasil

2. Professora da Faculdade de Fisioterapia e Terapia Ocupacional da Universidade Federal do Pará- UFPA. Belém, Pará, Brasil.

3. Professora da Universidade do Estado do Pará - UEPA. Belém, Pará, Brasil.

4. Professor da Faculdade Inspirar. Florianópolis, Santa Catarina, Brasil.
} 
A incontinência urinária (IU) ocorre comumente durante o ciclo gravídico-puerperal ${ }^{1}$. A IU inicia-se frequentemente na gestação e permanece no puerpério. Presença de IU na gestação, multiparidade, idade gestacional no parto maior ou igual a 37 semanas e constipação são fatores de risco para IU no puerpério ${ }^{2}$. A prevalência de IU é de até $75,25 \%$ na gravidez ${ }^{3}$ e de $37,9 \%$, no puerpério 4 .

Mulheres após o parto vaginal tem maior risco de incontinência urinária de esforço (IUE) pós-parto do que aqueles após cesárea ${ }^{5}$. De fato, a IUE persistente é mais frequente em mulheres que tiveram parto vaginal do que nas de parto cesárea, contudo mulheres que sofreram de IUE durante a gravidez tendem a se recuperar no primeiro ano pós-parto ${ }^{6}$.

A cesariana é uma intervenção cirúrgica que tem papel importante na diminuição da morbidade e mortalidade materna e perinatal; quando desnecessária, expõe a gestante a riscos, em geral maiores do que no parto vaginal ${ }^{7}$. Umas das razões pela opção de cesariana seria a prevenção de efeitos adversos sobre as estruturas do assoalho pélvico, tais como prolapsos, IU e incontinência fecal, normalmente associados ao parto vaginal ${ }^{8}$. O medo de lesões no canal vaginal, provocando a dor durante as relações sexuais, também é uma razão relevante para as mulheres ${ }^{9}$. No entanto, o parto cesáreo pode prevenir tais danos ao assoalho pélvico somente nos primeiros meses do pós-parto, sugerindo que, mais do que o parto, o período gestacional é responsável pelos danos no assoalho pélvico, devido as alterações anatômicas e funcionais decorrentes da gravidez, o que desencadearia problemas no trato urinário inferior, responsáveis pelos desagradáveis sintomas urinários (SU) ${ }^{10,11}$. Assim, os SU estão presentes nas mulheres independentemente da via de parto $\mathrm{SU}^{12}$.

A paridade parece envolver processos que predispõem o surgimento dos SU, de forma que a frequência desses sintomas em nulíparas é de $10,1 \%$, nas mulheres submetidas à cesárea de $15,9 \%$, e nas que tiveram parto normal de $21,0 \%{ }^{13}$.

Contudo, ainda não se tem certeza quais sintomas estão relacionados a cada tipo de parto. A fim de determinar quais os SU são mais frequentes nas puérperas, esta pesquisa teve como objetivo comparar os sintomas urinários de primíparas de parto normal e cesárea.

\section{MATERIAL E MÉTODOS}

Trata-se de um estudo observacional analítico do tipo transversal, realizado segundo as Normas de Pesquisa Envolvendo Seres Humanos do Conselho Nacional de Saúde (Res. CNS n. 466/12). O estudo teve início após a aprovação do Comitê de Ética em Pesquisa da Universidade da Amazônia, conforme o Parecer 655.640.

O estudo ocorreu no Centro de Saúde Escola do Marco, da Universidade do Estado do Pará, na Cidade de Belém do Pará, com mulheres que fizeram o acompanhamento do pré-natal nos anos de 2012 e 2013. A amostra foi definida por conveniência.

Os critérios de inclusão para a participação da pesquisa foram: mulheres com idade entre 18 e 35 anos, primíparas de parto normal ou cesárea no período de dois meses a dois anos do pós-parto e que tenham assinado o TCLE. E os critérios de exclusão foram: mulheres multíparas, que tiveram histórico de gravidez de risco, diabéticas, hipertensas, que estivessem em novo período gestacional ou tiveram período gestacional menor que 37 semanas, e que apresentaram algum sintoma urinário prévio à gravidez.

No período de outubro de 2012 a dezembro de 2013, 141 mulheres realizaram o pré-natal no Centro de Saúde Escola do Marco. As mulheres foram contatadas por telefone e convidadas a participar da pesquisa. As que aceitaram foram direcionadas para o Centro para a coleta de dados, durante os meses de junho e julho de 2014.

Para a coleta foi aplicado um questionário individual pelas pesquisadoras, que o preenchiam a partir das respostas das voluntárias. Para a coleta de dados foi construído um questionário pelas pesquisadoras baseado no estudo de Souza ${ }^{14}$, que foi direcionado a gravidas de baixo risco para identificar os sintomas urinários.

A análise dos dados foi feita com aplicação do teste exato de Fisher pelo programa SPSS 20.0, com nível de significância de 5\%.

\section{RESULTADOS}

Participaram do estudo 98 mulheres primíparas. Dentre os SU estudados na pesquisa, como a noctúria; polaciúria; urgência; urge-incontinência e enurese, relacionados ao tipo de parto, os resultados apontaram que as $41(41,84 \%)$ primíparas de parto normal têm maior chance de apresentarem algum sintoma urinário, em relação as 57 (58,16\%) mulheres que realizaram parto cesárea.

Quanto à prevalência dos SU relacionados ao tipo de parto, observou-se que a noctúria teve frequência maior nas mulheres que realizaram parto cesárea $(22,81 \%)$, enquanto que a urge-incontinência teve frequência maior nas mulheres que tiveram parto normal $(31,71 \%)$. Quando relacionado os SU com a paridade, utilizando o teste exato de Fisher com significância de 5\%, observou-se que o valor do "p" é maior que o nível de significância. Portanto, o surgimento dos SU independe da via de parto, conforme representado na Tabela 1. 
Tabela 1: Distribuição absoluta e percentual e comparação dos SU em primíparas $(n=57)$ de parto cesárea e normal. Belém, Pará, 2014.

\begin{tabular}{|c|c|c|c|c|c|c|c|c|c|c|}
\hline \multirow{3}{*}{ Sintomas } & \multicolumn{4}{|c|}{ Cesárea } & \multirow{3}{*}{ P valor* } & \multicolumn{4}{|c|}{ Normal } & \multirow{3}{*}{ P valor* } \\
\hline & \multicolumn{2}{|c|}{ Sim } & \multicolumn{2}{|c|}{ Não } & & \multicolumn{2}{|c|}{ Sim } & \multicolumn{2}{|c|}{ Não } & \\
\hline & $n$ & $\%$ & $\mathrm{n}$ & $\%$ & & $n$ & $\%$ & $\mathbf{n}$ & $\%$ & \\
\hline Noctúria & 13 & 22,8 & 44 & 77,2 & 0,194 & 13 & 31,7 & 28 & 68,3 & 0,273 \\
\hline Polaciúria & 8 & 14,0 & 49 & 85,9 & 0,321 & 6 & 14,6 & 35 & 85,3 & 0,447 \\
\hline Urge-incontinência & 3 & 5,2 & 54 & 94,7 & 0,225 & 5 & 12,2 & 36 & 87,8 & 0,360 \\
\hline Urgência & 3 & 5,2 & 54 & 94,7 & 0,578 & 4 & 9,7 & 37 & 90,2 & 1,000 \\
\hline Enurese & 1 & 1,8 & 56 & 98,3 & 0,664 & 1 & 2,5 & 40 & 97,5 & 1,000 \\
\hline
\end{tabular}

* Teste exato de Fisher.

A Razão de Prevalência mostrou que primíparas de parto normal tem um risco de pelo menos 2,3 vezes maior de apresentar a urgência miccional, 1,8 vezes a urge-incontinência, 1,04 vezes a polaciúria e 1,4 vezes a noctúria e a enurese, em relação às mulheres que fizeram o parto cesárea.

\section{DISCUSSÃO}

Este estudo teve por objetivo comparar os SU de primíparas de parto normal e cesárea. Observou-se que os SU mais frequentes em primíparas de parto cesárea foi a noctúria, seguida da polaciúria, enquanto que nas primíparas de parto normal os sintomas mais comuns foram a urge-incontinência e urgência miccional. Este estudo corrobora com a literatura que tem mostrado que mulheres os sintomas noctúria, a polaciúria, a urgência e a urge-incontinência são os sintomas mais frequentes em primiparas ${ }^{15-17}$.

Nesta pesquisa encontrou-se que as primíparas de parto normal têm risco maior de desenvolver os $\mathrm{SU}$ em relação as de pós-parto cesárea. A relação dos SU com a via de parto já foi investigada anteriormente em nulíparas, primíparas, multíparas e revelou que as nulíparas apresentam menos sintomas do que as primíparas e as multíparas apresentam mais sintomas do que as nulíparas e primíparas ${ }^{18}$, sendo que após parto vaginal existe maior propensão a algum SU do que após o parto cesariano ${ }^{19,20}$.

Os informes fornecidos pela literatura mostram que os SU se devem às modificações da troficidade muscular nos períodos de alteração hormonal durante a gestação, e ao traumatismo obstétrico. Em função disso, sugere-se que a gestação desempenha um papel importante para o surgimento do SU, independentemente do tipo de parto ${ }^{21}$.

Logo, os achados do presente estudo apontam que tanto as mulheres que têm parto normal ou cesárea podem vir a apresentar SU de urgência miccional, urge-incontinência, noctúria, polaciúria e enurese, independentemente do tipo de parto. No entanto, existe evidência de que os SU desencadeados pela gravidez cessam no período puerperal tardio, seja o parto normal ou cesárea ${ }^{22}$.

O parto vaginal foi associado como principal fator do surgimento dos SU em primíparas, em virtude dos danos que podem provocar à integridade da musculatura e inervação do assoalho pélvico ${ }^{23}$. Por outro lado, foi comprovado que o surgimento dos SU no pós-parto vaginal imediato de primíparas é normal, porém estatisticamente esse risco não é significante ${ }^{24}$.

As primíparas submetidas à cesariana ficaram protegidas do desenvolvimento do SU após quatro meses do pós-parto, se comparadas às que foram submetidas ao parto vaginal ${ }^{25}$, embora não haja evidências conclusivas de que a cesariana diminui o risco do surgimento de disfunções miccionais quando precedida de trabalho de parto ${ }^{26}$.

Da mesma forma, 125 primíparas no pós-parto também não apresentaram diferença estatística significante dos SU, não havendo associação positiva com o tipo de parto ${ }^{27}$. Isto corrobora com o presente estudo, cujos resultados não apresentaram correlação direta da via de parto com o surgimento dos SU, evidenciando que ambos os tipos podem apresentar distúrbios miccionais.

Os resultados deste estudo sugerem que 
as vias de parto não foram fatores de risco determinantes para a disfunção do trato urinário inferior após o parto, representados pelos sintomas urinários irritativos. Essa informação poderá contribuir nas decisões das gestantes concernentes a seu parto. Contudo, existe a necessidade de realizar outras pesquisas relacionadas ao tema para contribuir com a comunidade científica sobre os SU em primíparas de parto normal e cesárea.

\section{REFERÊNCIAS}

1. Sangsawang B, Sangsawang N. Stress urinary incontinence in pregnant women: a review of prevalence, pathophysiology, and treatment. Int Urogynecol J. 2013;24:901e12.

2. Saboia DM, Bezerra $\mathrm{K}$ de $\mathrm{C}$, Vasconcelos Neto JA, Bezerra LRPS, Oriá MOB, Vasconcelos CTM. Eficácia das intervenções realizadas no pós-parto para prevenir incontinência urinária: revisão sistemática. Rev. Bras. Enferm. 2018; 71 ( Suppl 3 ): 1460-1468.

3. Nigam A, Ahmad A, Gaur D, Elahi AA, Batra S. Prevalence and risk factors for urinary incontinence in pregnant women during late third trimester. Int J Reprod Contracept Obstet Gynecol. 2017;26:5(7):2187-91.

4. MacArthur C, Wilson D, Herbison P, Lancashire RJ, Hagen $\mathrm{S}$, Toozs-Hobson $\mathrm{P}$, et al. Urinary incontinence persisting after childbirth: extent, delivery history, and effects in a 12year longitudinal cohort study. BJOG. 2016;123(6):1022-9.

5. Kokabi R, Yazdanpanh D. Effects of delivery mod and sociodemographic factors on postpartum stress urinary incontinence in primipara women: a prospective cohort study. J Chin Med Assoc 2017:80:498e502.

6. Lin YH, Chang SD, Hsieh WC, Chang YL, Chueh HY, Chao AS, et al. Persistent stress urinary incontinence during pregnancy and one year after delivery; its prevalence, risk factors and impact on quality of life in Taiwanese women: An observational cohort study. Taiwan J Obstet Gynecol. 2018 Jun;57(3):340-345

7. Barbosa AMP, Carvalho LR, Martins AMVC et al. Efeito da via de parto sobre a força muscular do assoalho pélvico. Rev. Bras. Ginecol. Obstet. 2005; 27(11): 677-82.

8. Dietz HP, Schierlitz L. PelvicFloor Trauma in ChildbirthMythor Reality? Aust N Z J Obstet Gynaecol. 2005; 45(1): 3-11.

9. Griboski RA, Guilhem D. Mulheres e profissionais de saúde: o imaginário cultural na Humanização ao parto e nascimento. Texto Contexto Enfermagem. 2006; 15(1): 107-14.

10. Scarpa KP, Hermann V, Palma PCR, Riccetto CLZ, Morais SS. Sintomas urinários irritativos após parto vaginal ou cesárea. Rev. Assoc. Med. Bras. 2009; 55(4): 416-420.

11. Rortveit GMD, Anne KD PHD, Yngvild SH et al. Urinary Incontinence after Vaginal Delivery or Cesarean Section. N Engl J Med 2003; 101(6)348:900-907.

12. Moisés ECD, Brito LGO, Duarte G, Freitas MMS Disfunções miccionais no período gestacional e puerperal. Femina. 2011; 39(8): 409-412.

13. Rortveit G, Daltveit AK, Hunskaar S; Norwegian Epincont Study. Urinary incontinence after vaginal delivery or cesarean section. N Engl J Med. 2003; 348(10): 900-907 Rortveit G, Daltveit AK, Hunskaar S; Norwegian Epincont Study. Urinary incontinence after vaginal delivery or cesarean section. N Engl J Med. 2003; 348(10): 900-907.

14. Souza APP. Prevalência dos sintomas urinários na gestação de baixo risco em usuários do Sistema Único de

\section{CONCLUSÃO}

Nesta pesquisa encontrou-se que o surgimento dos sintomas urinários ocorreu, independentemente, da via de parto. A urge-incontinência foi a mais frequente nas primíparas de parto normal e a noctúria, o sintoma mais frequente nas primíparas de parto cesárea.

Saúde (SUS) [Dissertação]. Rio de Janeiro: Universidade Estácio de Sá; 2008.

15. Scarpa KP, Herrmann V, Palma PCR et al. Prevalência de sintomas urinários no terceiro trimestre da gestação. Rev. Assoc. Med. Bras. 2006; 52(3): 153-155.

16. Van Brummen HJ, Bruinse HW, Van Der Bom JG et al Hou do the prevalences of urogenital symptams changes during pregnancy? Neuronal urodynam. 2006; 25(2): 135-9.

17. Frederice CP. Assoalho pélvico e sintomas urinários na gestação e após o parto. [Dissertação]. São Paulo: Universidade Estadual de Campinas - UNICAMP, 2010.

18. Palma T, Raimondi M, Souto S, Fozzatti C, Palma P, Riccetto C.. Prospective study of prevalence of overactive bladder symptoms and child-bearing in women of reproductive age. J Obstet Gynaecol Res. 2013;39(8):1324-9.

19. Handa VL, Harvey L, Fox HE, Kjerulff KH. Parity and route of delivery: does cesarean delivery reduce bladder symptoms later in life? Am J Obstet Gynecol. 2004;191(2):4639.

20. Sermin T-T, Nezihe K, Beji EA, Önay Y. Determining lower urinary tract symptoms and associated risk factors in young women. Gynecology Obstetrics. 2012; 118(1): 27-30.

21. Lopes DBM, Praça NS. Incontinência Urinária autorreferida no pós-parto. Texto e Contexto de Enferma, Florianópolis, 2010; 19(4):667-74.

22. Pereira SB. Impacto do parto na atividade eletromiográfica do assoalho pélvico e nos sintomas do trato urinário inferior: estudo prospectivo e comparativo [tese]. São Paulo: Universidade Estadual de Campinas - UNICAMP, 2008.

23. Panayi DC, Krullar V. Urogynaecological problems in pregnancy and postpartum sequelae. Current opinion in obstetric and gynecology. 2009; 21(1): 97-100.

24. Boyles SH, Li H, Mori T, Osterweil P, Guise JM. Effect of mode of delivery on in the incidence of urinary incontinence in primiparous women. Obstet Gynecol. 2009;113(1):134-41.

25. Eftekhar T, Hajibaratali B, Ramezanzadeh F, Shariat M Postpartum evoluation of stress urinary incontinence among primíparas. Int J Gynaecol Obstet. 2006;94(2):1148

26. Madill JS, Mclean LA. Contextual model of pelvic floor muscle defects in female stress urinary incontinence. Ann N Y Acad Sci. 2007;1101:335-60.

27. Valeton $\mathrm{CT}$. Avaliação da incontinência urinária no terceiro trimestre de gestação e no puerpério em mulheres do programa mãe curitibana. Curitiba: Pontíficia Universidade Católica do Paraná; 2010.

Correspondência

Nome completo: Erica Feio Carneiro Nunes

Endereço: Tv. Perebebuí, 2623

CEP: $66087-670$

Cidade: Belém - Pará - Brasil

E-mail: erica@perineo.net 